Mitteilungen der Österreichischen Geographischen Gesellschaft, 159. Jg. (Jahresband), Wien 2017, S. 379-392

\title{
11. Konferenz der Vereinten Nationen Zur STANDARdisierung GeOgraphischer NAMEN UND 30. Sitzung der Expertengruppe der Vereinten Nationen für geographische Namen, New York
}

\author{
Peter JORDAN, Wien*
}

mit 5 Abb. und 1 Tab. im Text

\section{INHALT}

1 Blick zurück und nach vorn .................................................................................................. ??

2 Österreichische Beiträge ........................................................................................................ ???

3 Umschrift des Arabischen ................................................................................................ ??

4 „Side events“, Funktionswechsel und Ehrungen ...................................................................... ???

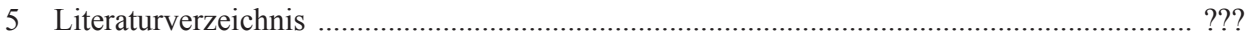

\section{Blick zurück und nach vorn}

Die 11. Konferenz der Vereinten Nationen zur Standardisierung geographischer Namen $\left(11^{\text {th }}\right.$ United Nations Conference on the Standardisation of Geographical Names, UNCSGN), umrahmt von je einem Tag der 30. Sitzung der Expertengruppe der Vereinten Nationen für geographische Namen (United Nations Group of Experts on Geographical Names, UNGEGN), stand ganz im Zeichen zweier Blickrichtungen: (1) aus Anlass des 50-jährigen Jubiläums der Konferenzen und der Expertengruppe eines Blicks zurück auf eine stolze Leistungsbilanz und (2) eines Blicks nach vorn in Gestalt einer umfassenden Diskussion über eine grundlegende Änderung der Arbeitsweise dieser beiden Einrichtungen. Vor allem Zweiteres gab den beiden Veranstaltungen im Hauptquartier der Vereinten Nationen am East River in New York (Abb. 1) den Charakter einer Zäsur.

Das 50-Jahr-Jubiläum wurde von der UNGEGN-Leitung und von etlichen Divisions (im Sinne von nach sprachlichen und/oder regionalen Gesichtspunkten als Untereinheiten der UNGEGN zusammenarbeitenden Ländergruppen), natürlich besonderes von jenen, die als erste eingerichtet worden waren, zu Rückblicken und Leistungsbilanzen genützt. Von den heute bestehenden 24 Divisions (Einige bestanden nur zeitweilig und wurden wieder aufgelöst.) waren die folgenden (in der Reihenfolge des englischen Alphabets) von Anfang an und bis heute aktiv: Arabic Division, Asia East (other than China) Division, Asia South-West (other than Arabic) Division, Dutch- and

\footnotetext{
* Hofrat Prof. h.c. Univ.-Doz. Dr. Peter Jordan, Institut für Stadt- und Regionalforschung, Österreichische Akademie der Wissenschaften; Research Fellow, University of the Free State Bloemfontein, South Africa; Postgasse 7/4/2, A-1010 Wien; E-Mail: peter.jordan@oeaw.ac.at, http://www.oeaw.ac.at/isr
} 


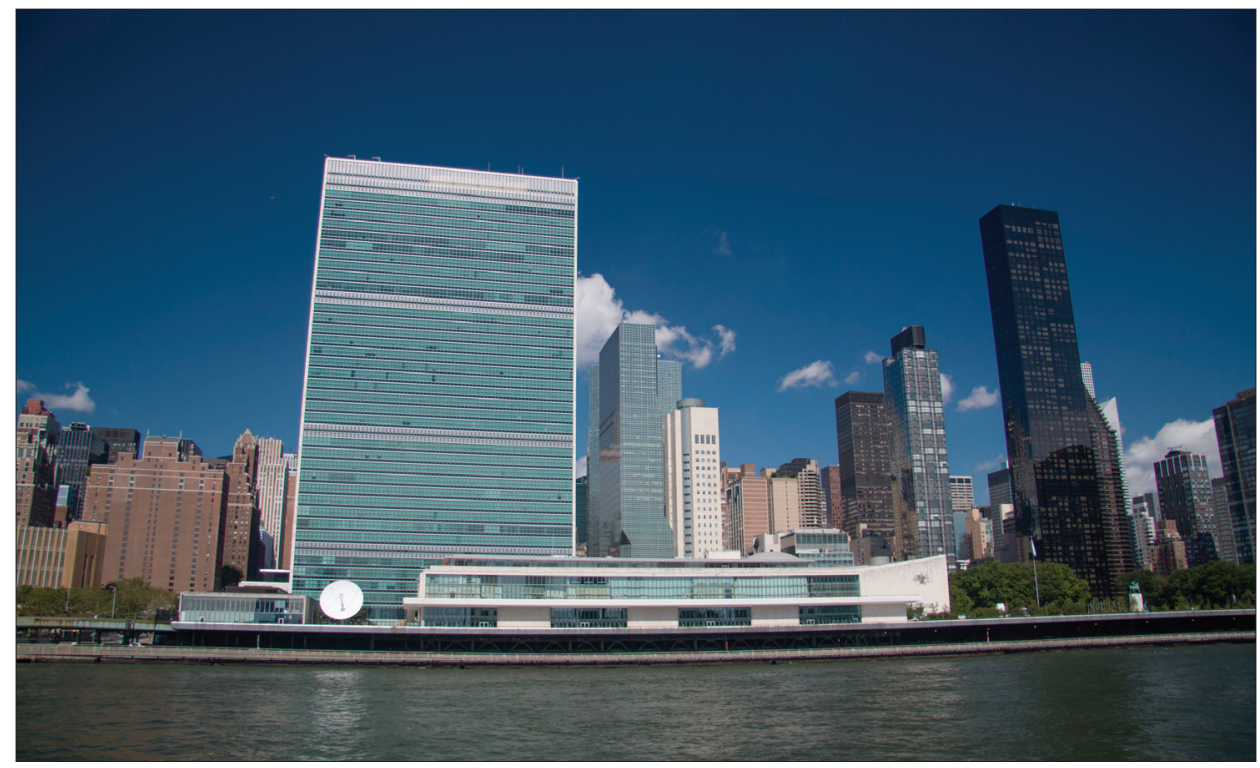

Foto: $\quad$ Maciej ZYCH 2017

Abb. 1: Das Hauptquartier der Vereinten Nationen am East River, New York

German-speaking Division (DGSD), East-Central and South-East Europe Division, India Division, Latin America Division, Norden Division, Romano-Hellenic Division, United Kingdom Division, United States of America/Canada Division.

Auch für die Niederländisch-deutschsprachige Gruppe (Dutch- and German-speaking Division, DGSD), der neben Deutschland, der Schweiz, den Niederlanden, Belgien, Suriname und Südafrika auch Österreich angehört, war dies der Anlass, einen Rückblick zu geben. Dabei wurde besonders der Persönlichkeiten gedacht, die aus dem Kreis der DGSD die UNGEGN mitgegründet und/oder in ihr und den Konferenzen eine maßgebliche Rolle gespielt haben und heute nicht mehr leben oder aktiv sind: Rolf BöHME (Deutschland, 1917-1997), Josef BREU (Österreich, 1914-1998), Erfried HAACK (früher DDR, dann Deutschland, 1929-), Emil Meynen (Deutschland, 1902-1994), Fer Ormeling sen. (Niederlande, 1912-2002), Peter E. RaPer (Südafrika) und Jörn Sievers (Deutschland, 1942-).

Selbst aus dieser Reihe ragt der Österreicher Josef BREU (Abb. 2) noch heraus, denn er gehörte nicht nur zu den Gründern der UNGEGN, sondern war von 1979 bis 1982 auch deren Vorsitzender, initiierte mit den Toponymic Guidelines for Map and Other Editors for International Use ein grundlegendes UNGEGN-Projekt, engagierte sich maßgeblich in den Bereichen der Umschriftsysteme und der Exonyme, richtete in Österreich nach den Empfehlungen der Vereinten Nationen mit der Arbeitsgemeinschaft für Kartographische Ortsnamenkunde (AKO) ein landesweites Expertengremium ein und gab ein geographisches Namenbuch von Österreich heraus (BREU 1975), das ebenfalls nach den Empfehlungen der Vereinten Nationen gestaltet war und das für viele andere Länder zum Muster wurde.

Tabelle 1 gibt einen Überblick über Präsenz und Rolle Österreichs bei den Konferenzen der Vereinten Nationen zur Standardisierung geographischer Namen (UNCSGN) und den UNGEGN-Sitzungen in dieser Zeitspanne eines halben Jahrhunderts. 


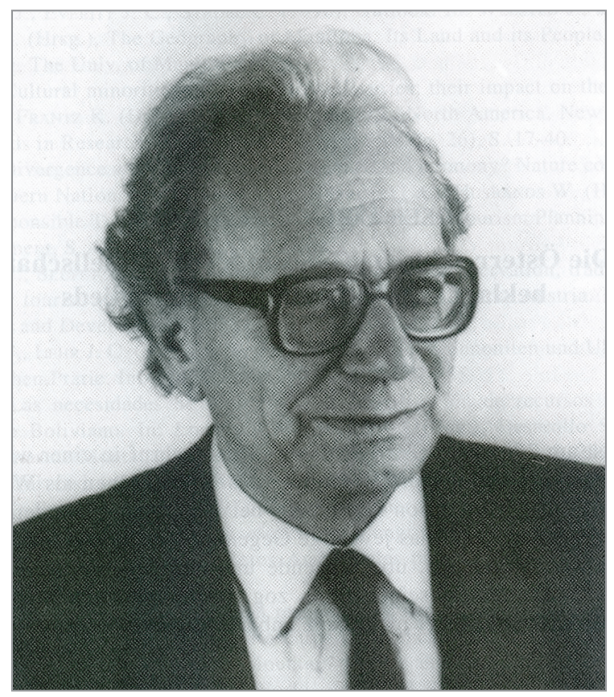

Abb. 2: Josef Breu 1914-1998

Das zweite markante und für die Zukunft wohl bestimmende Ereignis dieser Konferenz war der Beschluss über die neue Organisationsform der toponymischen Aktivitäten der Vereinten Nationen. Er war notwendig geworden, weil sich vor fünf Jahren mit dem United Nations Committee of Experts on Global Geospatial Information Management (UN-GGIM) ein Expertengremium der Vereinten Nationen etabliert hatte, das sich mit der Gesamtheit raumbezogener Informationen beschäftigt, darunter auch mit geographischen Namen. Sowohl UNGEGN als auch UN-GGIM unterstehen dem Economic and Social Council (ECOSOC) der Vereinten Nationen, der Interesse an einer guten Kooperation zwischen beiden Gremien und möglichst geringen Gesamtkosten hat. UN-GGIM hält jährliche Sitzungen ab und verabschiedet dabei Resolutionen. Wenn die UNGEGN wie bisher nur alle fünf Jahre auf Konferenzen (UNCSGN) Resolutionen verabschieden könnte, geriete sie zeitlich ins Hintertreffen und die Kooperation litte darunter.

Daher wurde auf der diesjährigen Konferenz von der erweiterten UNGEGN-Leitung (unter Einschluss der Working Group Convenors) nach langen Diskussionen der Vorschlag vorgelegt, Konferenzen und Sitzungen zeitlich und funktional zusammenzulegen und im Abstand von zwei Jahren durchzuführen. Diese Sitzungen der Expertengruppe hätten nun auch die Kompetenz von Konferenzen und könnten auch Resolutionen beschließen, die dann nur noch vom ECOSOC bestätigt werden müssten. Befürchtungen, solche Sitzungen wären dann keine Expertentreffen mehr, sondern würden zunehmend von Diplomaten beschickt und von politischen Interessen beherrscht, mündeten also in einen Rückzug der Wissenschaft (v.a. Geographie, Kartographie, Geodäsie, Linguistik) aus der UNGEGN, blieben zwar bestehen. Bill WATT, UNGEGN Chair (Australien), konnte aber überzeugend darlegen, dass die Kooperationsfähigkeit mit UN-GGIM das primäre und für die UNGEGN überlebenswichtige Ziel sei und erreichte so letztlich ein fast einstimmiges Votum für das neue Organisationsmodell.

Allerdings stieß die Ausgangsfassung der Resolution doch auf viele Widerstände, besonders der Republik Korea, deren Diplomaten jede Gelegenheit zu Einwänden nützten. Es kam daher im Plenum und (noch mehr) außerhalb zu etlichen Verhandlungsrunden, bis eine Endfassung des Dokuments akkordiert werden konnte. Sie enthielt (wegen der Einwände) keine Details wie den 
Peter Jordan

\begin{tabular}{|c|c|c|}
\hline Konferenz / Sitzung & Vertreter Österreichs & Internationale Funktion \\
\hline $\begin{array}{l}\text { 1. UNCSGN, Genf, } \\
\text { 4.-22. September } 1967\end{array}$ & Josef Breu & - \\
\hline $\begin{array}{l}\text { 1. UNGEGN-Sitzung, Genf, } \\
\text { 22. September } 1967\end{array}$ & Josef BREU & - \\
\hline $\begin{array}{l}\text { 2. UNGEGN-Sitzung, New York, } \\
\text { 10.-20. März } 1970\end{array}$ & Josef BREU & - \\
\hline $\begin{array}{l}\text { 3. UNGEGN-Sitzung, New York, } \\
\text { 2.-12. Februar } 1971\end{array}$ & Josef BREU & - \\
\hline $\begin{array}{l}\text { 4. UNGEGN-Sitzung, London, } \\
\text { 9. Mai und 1. Juni } 1972\end{array}$ & Josef BREU & - \\
\hline $\begin{array}{l}\text { 2. UNCSGN, London, } \\
\text { 10.-31. Mai } 1972\end{array}$ & - & - \\
\hline $\begin{array}{l}\text { 5. UNGEGN-Sitzung, New York, } \\
\text { 5.-16. März } 1973\end{array}$ & - & - \\
\hline $\begin{array}{l}\text { 6. UNGEGN-Sitzung, New York, } \\
\text { 5.-26. März } 1975\end{array}$ & Josef BreU & - \\
\hline $\begin{array}{l}\text { 7. UNGEGN-Sitzung, Athen, } \\
\text { 16. August und 8. September } 1977\end{array}$ & Josef BreU & - \\
\hline $\begin{array}{l}\text { 3. UNCSGN, Athen, } \\
\text { 17. August - 7. September } 1977\end{array}$ & Josef BreU & - \\
\hline $\begin{array}{l}\text { 8. UNGEGN-Sitzung, New York, } \\
\text { 26. Februar - 9. März } 1979\end{array}$ & Josef BreU & UNGEGN Chair \\
\hline $\begin{array}{l}\text { 9. UNGEGN-Sitzung, New York, } \\
\text { 17.-27. Februar } 1981\end{array}$ & Josef BREU & UNGEGN Chair \\
\hline $\begin{array}{l}\text { 10. UNGEGN-Sitzung, Genf, } \\
\text { 23. August und 15. September } 1982\end{array}$ & Josef BreU & $\begin{array}{l}\text { Coordinator, Toponymic Guide- } \\
\text { lines for Map and Other Editors } \\
\text { for International Use }\end{array}$ \\
\hline $\begin{array}{l}\text { 4. UNCSGN, Genf } \\
\text { 24. August - 14. September } 1982\end{array}$ & Josef BreU & - \\
\hline $\begin{array}{l}\text { 11. UNGEGN-Sitzung, Genf, } \\
\text { 15.-23. Oktober } 1984\end{array}$ & Josef BREU & $\begin{array}{l}\text { Coordinator, Toponymic Guide- } \\
\text { lines for Map and Other Editors } \\
\text { for International Use }\end{array}$ \\
\hline $\begin{array}{l}\text { 12. UNGEGN-Sitzung, Genf, } \\
\text { 29. September - 7. Oktober } 1986\end{array}$ & Peter JoRdan & DGSD Chair \\
\hline $\begin{array}{l}\text { 13. UNGEGN-Sitzung, Montreal, } \\
\text { 17. und } 31 \text {. August } 1987\end{array}$ & Josef BreU & $\begin{array}{l}\text { Co-convenor, Working Group on } \\
\text { a Single Romanization System for } \\
\text { Each Non-Roman Writing System; } \\
\text { Coordinator, Toponymic Guide- } \\
\text { lines for Map and Other Editors } \\
\text { for International Use; } \\
\text { DGSD Chair }\end{array}$ \\
\hline
\end{tabular}




\begin{tabular}{|c|c|c|}
\hline Konferenz / Sitzung & Vertreter Österreichs & Internationale Funktion \\
\hline $\begin{array}{l}\text { 5. UNCSGN, Montreal, } \\
\text { 18.-30. August } 1987\end{array}$ & Josef Breu & $\begin{array}{l}\text { Vice-Chair, Technical Committee } \\
\text { III }\end{array}$ \\
\hline $\begin{array}{l}\text { 14. UNGEGN-Sitzung, Genf, } \\
\text { 17.-26. Mai } 1989\end{array}$ & - & - \\
\hline $\begin{array}{l}\text { 15. UNGEGN-Sitzung, Genf, } \\
\text { 11.-20. November } 1991\end{array}$ & Peter JoRDAN & - \\
\hline $\begin{array}{l}\text { 16. UNGEGN-Sitzung, New York, } \\
\text { 24. August und 4. September } 1992\end{array}$ & Helmut Desoye & - \\
\hline $\begin{array}{l}\text { 6. UNCSGN, New York, } \\
\text { 25. August - 3. September } 1992\end{array}$ & Helmut Desoye & - \\
\hline $\begin{array}{l}\text { 17. UNGEGN-Sitzung, New York, } \\
\text { 13.-24. Juni } 1994\end{array}$ & Isolde HAUSNER & $\begin{array}{l}\text { Coordinator, Toponymic Guide- } \\
\text { lines for Map and Other Editors } \\
\text { for International Use; } \\
\text { DGSD Chair }\end{array}$ \\
\hline $\begin{array}{l}\text { 18. UNGEGN-Sitzung, Genf, } \\
\text { 12.-23. August } 1996\end{array}$ & - & \\
\hline $\begin{array}{l}\text { 19. UNGEGN-Sitzung, New York, } \\
\text { 12. und 23. Jänner } 1998\end{array}$ & Isolde HAUSNER & $\begin{array}{l}\text { Coordinator, Toponymic Guide- } \\
\text { lines for Map and Other Editors } \\
\text { for International Use }\end{array}$ \\
\hline $\begin{array}{l}\text { 7. UNCSGN, New York, } \\
\text { 13.-22. Jänner } 1998\end{array}$ & Isolde HAUSNER & - \\
\hline $\begin{array}{l}\text { 20. UNGEGN-Sitzung, New York, } \\
\text { 17.-28. Jänner } 2000\end{array}$ & Isolde HAUSNER & $\begin{array}{l}\text { Coordinator, Toponymic Guide- } \\
\text { lines for Map and Other Editors } \\
\text { for International Use }\end{array}$ \\
\hline \multirow[t]{2}{*}{$\begin{array}{l}\text { 21. UNGEGN-Sitzung, Berlin, } \\
\text { 26. August und 6. September } 2002\end{array}$} & Isolde HAUSNER & $\begin{array}{l}\text { Coordinator, Toponymic Guide- } \\
\text { lines for Map and Other Editors } \\
\text { for International Use }\end{array}$ \\
\hline & Peter JORDAN & - \\
\hline \multirow{3}{*}{$\begin{array}{l}\text { 8. UNCSGN, Berlin, } \\
\text { 27. August - 5. September } 2002\end{array}$} & Isolde HAUSNER & - \\
\hline & Markus Lutterotti & - \\
\hline & Peter JoRDAN & - \\
\hline \multirow[t]{2}{*}{$\begin{array}{l}\text { 22. UNGEGN-Sitzung, New York, } \\
\text { 20.-29. April } 2004\end{array}$} & Isolde HAUSNER & $\begin{array}{l}\text { Coordinator, Toponymic Guide- } \\
\text { lines for Map and Other Editors } \\
\text { for International Use }\end{array}$ \\
\hline & Peter JoRDAN & $\begin{array}{l}\text { Co-convenor, Working Group on } \\
\text { Exonyms }\end{array}$ \\
\hline
\end{tabular}




\begin{tabular}{|c|c|c|}
\hline Konferenz / Sitzung & Vertreter Österreichs & Internationale Funktion \\
\hline \multirow[t]{3}{*}{$\begin{array}{l}\text { 23. UNGEGN-Sitzung, Wien, } \\
\text { 28. März - 4. April } 2006\end{array}$} & Isolde HAUSNER & $\begin{array}{l}\text { Coordinator, Toponymic Guide- } \\
\text { lines for Map and Other Editors } \\
\text { for International Use; } \\
\text { DGSD Chair }\end{array}$ \\
\hline & Peter JORDAN & $\begin{array}{l}\text { Convenor, Working Group on } \\
\text { Exonyms }\end{array}$ \\
\hline & $\begin{array}{l}\text { Sandra BARTHEL, } \\
\text { Hubert BERGMANN, } \\
\text { Lukas BIRSAK, Anita } \\
\text { CsENAR, Chris- } \\
\text { toph FINK, Michael } \\
\text { FuHRMANN, Georg } \\
\text { GARTNER, Markus } \\
\text { JoBST, Stoyan KoleV, } \\
\text { Johannes MAYER, } \\
\text { Stefan RopaC, Roman } \\
\text { STANI-FERTL, Johann } \\
\text { SEEDOCH, Gunter } \\
\text { STEFANITS, Leonhard } \\
\text { SuCHENWIRTH, Mihaly } \\
\text { SzABO, Josef VLASITS, } \\
\text { Rudolf VoIT }\end{array}$ & - \\
\hline \multirow[t]{3}{*}{$\begin{array}{l}\text { 24. UNGEGN-Sitzung, New York, } \\
\text { 20. und 31. August } 2007\end{array}$} & Peter JORDAN & $\begin{array}{l}\text { Convenor, Working Group on } \\
\text { Exonyms; } \\
\text { DGSD Chair }\end{array}$ \\
\hline & Isolde HAUSNER & $\begin{array}{l}\text { Coordinator, Toponymic Guide- } \\
\text { lines for Map and Other Editors } \\
\text { for International Use }\end{array}$ \\
\hline & Ulrike NGUYEN & - \\
\hline \multirow[t]{3}{*}{$\begin{array}{l}\text { 9. UNCSGN, New York, } \\
\text { 21.-30. August } 2007\end{array}$} & Peter JORDAN & $\begin{array}{l}\text { Vice-Chair, Technical Committee } \\
\text { III }\end{array}$ \\
\hline & Isolde HAUSNER & - \\
\hline & Ulrike NGUYEN & - \\
\hline \multirow[t]{3}{*}{$\begin{array}{l}\text { 25. UNGEGN-Sitzung, Nairobi, } \\
\text { 5.-12. Mai } 2009\end{array}$} & Peter JORDAN & $\begin{array}{l}\text { Convenor, Working Group on } \\
\text { Exonyms; } \\
\text { DGSD Chair }\end{array}$ \\
\hline & Hubert Bergmann & $\begin{array}{l}\text { Coordinator, Toponymic Guide- } \\
\text { lines for Map and Other Editors } \\
\text { for International Use }\end{array}$ \\
\hline & Mudau Rendani & - \\
\hline
\end{tabular}




\begin{tabular}{|c|c|c|}
\hline Konferenz / Sitzung & Vertreter Österreichs & Internationale Funktion \\
\hline \multirow[t]{3}{*}{$\begin{array}{l}\text { 26. UNGEGN-Sitzung, Wien, } \\
\text { 2.-6. Mai } 2011\end{array}$} & Peter JoRdAN & $\begin{array}{l}\text { Convenor, Working Group on } \\
\text { Exonyms; } \\
\text { DGSD Chair }\end{array}$ \\
\hline & Hubert BERGMANN & $\begin{array}{l}\text { Coordinator, Toponymic Guide- } \\
\text { lines for Map and Other Editors } \\
\text { for International Use }\end{array}$ \\
\hline & $\begin{array}{l}\text { Rainer DORMELS, } \\
\text { Michael DUSCHANEK, } \\
\text { Isolde HAUSNER, } \\
\text { Roman STANI-FERTL }\end{array}$ & - \\
\hline \multirow[t]{2}{*}{$\begin{array}{l}\text { 27. UNGEGN-Sitzung, New York, } \\
\text { 30. Juli und 10. August } 2012\end{array}$} & Peter JORDAN & $\begin{array}{l}\text { Convenor, Working Group on } \\
\text { Exonyms; } \\
\text { DGSD Chair }\end{array}$ \\
\hline & Hubert BergmanN & $\begin{array}{l}\text { Coordinator, Toponymic Guide- } \\
\text { lines for Map and Other Editors } \\
\text { for International Use }\end{array}$ \\
\hline \multirow{2}{*}{$\begin{array}{l}\text { 10. UNCSGN, New York, } \\
\text { 31. Juli - 9. August } 2012\end{array}$} & Peter JORDAN & Vice-Chair, Technical Committee III \\
\hline & Hubert BERGMANN & $\begin{array}{l}\text { Coordinator, Toponymic Guide- } \\
\text { lines for Map and Other Editors for } \\
\text { International Use }\end{array}$ \\
\hline \multirow[t]{2}{*}{$\begin{array}{l}\text { 28. UNGEGN-Sitzung, New York, } \\
\text { 28. April - 2. Mai } 2014\end{array}$} & Peter JORDAN & $\begin{array}{l}\text { Convenor, Working Group on } \\
\text { Exonyms }\end{array}$ \\
\hline & Gerhard RAMPL & $\begin{array}{l}\text { Coordinator, Toponymic Guide- } \\
\text { lines for Map and Other Editors for } \\
\text { International Use }\end{array}$ \\
\hline \multirow[t]{2}{*}{$\begin{array}{l}\text { 29. UNGEGN-Sitzung, Bangkok, } \\
\text { 25.-29. April } 2016\end{array}$} & Peter JORDAN & $\begin{array}{l}\text { Convenor, Working Group on } \\
\text { Exonyms }\end{array}$ \\
\hline & Gerhard RAMPL & $\begin{array}{l}\text { Coordinator, Toponymic Guide- } \\
\text { lines for Map and Other Editors for } \\
\text { International Use }\end{array}$ \\
\hline \multirow[t]{2}{*}{$\begin{array}{l}\text { 30. UNGEGN-Sitzung, New York, } \\
\text { 7. und 18. August } 2017\end{array}$} & Peter JoRdAN & $\begin{array}{l}\text { Convenor, Working Group on } \\
\text { Exonyms }\end{array}$ \\
\hline & Gerhard RAMPL & $\begin{array}{l}\text { Coordinator, Toponymic Guide- } \\
\text { lines for Map and Other Editors for } \\
\text { International Use }\end{array}$ \\
\hline \multirow{2}{*}{$\begin{array}{l}\text { 11. UNCSGN, New York, } \\
\text { 8.-17. August } 2017\end{array}$} & Peter JORDAN & Chair, Technical Committee III \\
\hline & Gerhard RAMPL & - \\
\hline
\end{tabular}

Quellen: Teilnehmerlisten und Berichte der UNCSGN und UNGEGN-Sitzungen

Tab. 1: Vertretung Österreichs bei UNCSGN and UNGEGN-Sitzungen 
Fortbestand der Gliederung der UNGEGN in Divisions mehr. Solche wurden in noch zu definierende und beschließende Verfahrensregeln (Rules of Procedure) ausgelagert. Der inhaltliche Kern des Vorschlags mit der Zusammenlegung der Funktionen von Konferenz und Sitzung konnte aber als Resolutionsentwurf beschlossen werden und wurde mittlerweile auch schon vom ECOSOC gebilligt. Es wird also von nun an keine Konferenzen der Vereinten Nationen zur Standardisierung geographischer Namen mehr geben, sondern nur noch Sitzungen, die aber auch die Kompetenz von Konferenzen haben. Die erste dieser Art ist für 29. April bis 3. Mai 2019 in New York anberaumt worden.

Die intensivere Zusammenarbeit zwischen UNGEGN und UN-GGIM wird zusätzlich auch Treffen zwischen Vertretern beider Gremien auf nationaler, kontinentaler und globaler Ebene erfordern, also im Falle Österreichs regelmäßige Besprechungen zwischen den österreichischen UNGEGN- und UN-GGIM-Delegierten, Sitzungen der Vertreter beider Gremien auf europäischer Ebene sowie die Teilnahme von UNGEGN-Vertretern an Sitzungen der UN-GGIM und umgekehrt. Das kann für ein nur in einem Verein (der Österreichischen Geographischen Gesellschaft, ÖGG) verankertes und mit der Österreichischen Akademie der Wissenschaften lediglich ,in Verbindung" stehendes Expertengremium wie die AKO, das auf den ehrenamtlichen Einsatz seiner Mitglieder angewiesen ist und ohne eigenes Budget auskommen muss, zeitlich und finanziell zu einer Herausforderung werden.

\section{2 Österreichische Beiträge}

Neben diesen beiden beherrschenden Themen brachten die insgesamt zwei Wochen dauernden, mit Delegationen aus 66 Ländern, Beobachtern diverser Organisationen und Institutionen, insgesamt 278 Personen, beschickten Tagungen aber auch sonst, besonders auch für Österreich wichtige Ergebnisse. Die vom österreichischen Bundesministerium für Europa, Integration und Äußeres delegierte und von der Österreichischen Akademie der Wissenschaften nominierte österreichische Delegation bestand aus dem Geographen und Kartographen Peter JoRDAN als Delegationsleiter und Convenor der UNGEGN Working Group on Exonyms sowie aus dem Linguisten Gerhard RAmPL als Mitglied der Delegation und UNGEGN-Koordinator der Toponymic Guidelines for Map and Other Editors for International Use.

Sie präsentierten im Plenum der Konferenz 9 der insgesamt 154 meritorischen Konferenzpapiere (Papiere rein organisatorischen Inhalts nicht mitgerechnet) und im Plenum der UNGEGN-Sitzung 3 der insgesamt 17 meritorischen Working Papers. ${ }^{1)}$ JoRDAN wurde zu Beginn der Konferenz, die unter dem Gesamtvorsitz von Ferjan Ormeling (Niederlande; Stellvertreter Brahim Atoui, Algerien, und Andreas HadjIRAftis, Zypern) ablief, auch zum Vorsitzenden des Technischen Komitees III gewählt, unter dessen Ägide im Plenum die Verhandlungen zu den Themen Exonyme, Umschriftsysteme, Aussprache und Staatennamen geführt wurden. Zu den Vorsitzenden der drei anderen Komitees wurden Annette TörensJö (Schweden, Technisches Komitee I), Pier-Giorgio Zaccheddu (Deutschland, Technisches Komitee II) und Leo Dillon (USA, Technisches Komitee IV) bestimmt. Gerhard RAMPL wurde zum Rapporteur-Assistenten des Technischen Komitees I bestellt.

Von den im Namen Österreichs vorgestellten Konferenzpapieren erfuhren die österreichischen Empfehlungen zur Verkehrsflächenbenennung die größte und eine äußerst positive Resonanz (JoRDAN 2017a). Vor allem die Delegationen der skandinavischen Länder und Kanadas, deren Vorbild

1) Alle Konferenzpapiere der UNCSGN und alle Working Papers der UNGEGN-Sitzung können auf der UNGEGN-Website (https://unstats.un.org/UNSD/geoinfo/UNGEGN/default.html) eingesehen werden. 
die von der AKO kurz zuvor beschlossenen und auf einer an der Universität Wien verfassten Diplomarbeit von Marlene KRAPF (KRAPF 2005) aufbauenden Empfehlungen in wesentlichen Punkten gefolgt waren, kommentierten sie mit großer Zustimmung. Die „Empfehlungen“ wurden bereits an die österreichischen Städte, den österreichischen Städtebund, den österreichischen Gemeindebund und die Gemeindebünde der österreichischen Länder versandt, seien aber auch hier in ihrer deutschen Fassung wiedergegeben:

Auf der Grundlage der Resolution VIII/2 der Vereinten Nationen empfiehlt die Arbeitsgemeinschaft für Kartographische Ortsnamenkunde (AKO) als das in Österreich für die Standardisierung geographischer Namen zuständige Expertengremium bei Verkehrsflächenbenennungen (Neubenennungen und Umbenennungen) die folgenden Kriterien zu beachten:

(1) Namen von Verkehrsfächen sollen in erster Linie die Orientierungsfunktion erfüllen.

(2) Gut eingeführte Namen sollen nicht ohne wichtigen Grund geändert werden.

(3) Bei Umbenennungen ist das Nachwirken des alten Namens im praktischen Gebrauch zu bedenken.

(4) Gleiche oder mit bestehenden leicht verwechselbare Namen innerhalb einer Gemeinde sind zu vermeiden.

(5) Bei Neubenennungen sollen Flurnamen und/oder andere lokal gebräuchliche Namen verwendet werden.

(6) Wenn doch Gedenknamen, d.h. Namen, die an Personen und Ereignisse erinnern, verwendet werden, soll dies mit Vorsicht und Zurückhaltung geschehen.

(a) Kommerzielle Namen, d.h. Namen von Firmen und ihren Produkten, sind zu vermeiden.

(b) Benennungen nach noch lebenden Personen sind zu vermeiden. Eine Interkalarfrist von mindestens fünf Jahren nach dem Tod der Person, nach der benannt wird, wird empfohlen.

(c) Die Person, nach der eine Verkehrsfläche benannt wird, soll zu diesem Ort Bezug oder (auch) für ihn Bedeutung gehabt haben (z.B. Geburtsort, Wirkungsstätte).

(d) Unter Bedachtnahme auf die Namenslänge sollen Namen nach Personen den Vor- und Familiennamen enthalten (z.B. Karl-Schweighofer-Gasse), um eine eindeutige Identifkation der Person zu ermöglichen. Die Verwendung von Titeln (Ing., Dr., Prof. etc.) ist zu vermeiden.

(e) In Anbetracht der historisch erklärbaren Asymmetrie von Benennungen nach Männern und Frauen wird empfohlen, Frauen bei Neubenennungen in besonderer Weise in Betracht zu ziehen.

Auch der Ständige Ausschuss für Geographische Namen (StAGN), das deutsche Pendant zur AKO, zugleich aber auch das Koordinationsorgan für die Standardisierung geographischer Namen aller deutschsprachigen Länder und Regionen, zieht nun in Erwägung, die österreichischen Empfehlungen zu Empfehlungen für alle StAGN-Länder zu machen, was ihnen sicher auch in Österreich weiteren Nachdruck verleihen wird.

Ein weiteres österreichisches, von Martina PІко-RUsTIA vom Urban-Jarnik-Institut in Klagenfurt a.W. verfasstes Konferenzpapier (PIKO-RUSTIA 2017) über die verdienstvollen Projekte der Sammlung, Dokumentation und Wiederbelebung (auch durch Wander- und Touristenkarten, Abb. 3) der slowenischen Haus- und Flurnamen in Kärnten wurde sehr beachtet - besonders auch deshalb, weil es sich gut in die Reihe der besonders in Kanada, Australien und Neuseeland, aber auch in China intensiven Bemühungen um die Erhaltung von geographischen Namen als kulturelles Erbe fügt (siehe dazu Cantile \& Kerfoot 2016). 


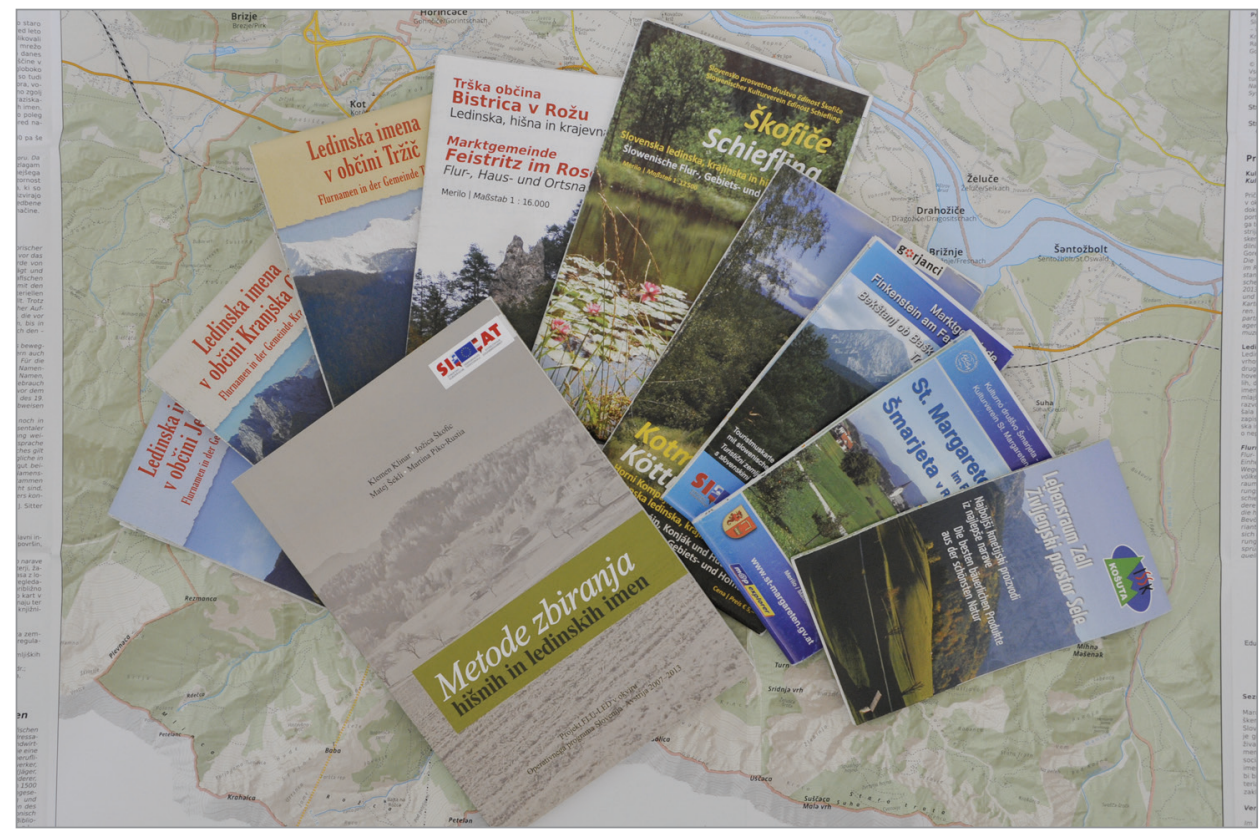

Foto: $\quad$ Martina PIKo-Rustia 2017

Abb. 3: Zweisprachig slowenisch-deutsche Wander- und Touristenkarten mit Hof- und Flurnamen von Südkärnten

Weitere im Namen Österreichs vorgestellte Konferenzpapiere behandelten den Exonymengebrauch in Österreich (JoRDAN 2017b), gaben eine Bilanz der UNGEGN Working Group on Exonyms nach 15 Jahren ihres Bestandes mit einer Liste der noch offenen Fragen (JORDAN 2017c) sowie einen Überblick über die namenkundlichen Aktivitäten in Österreich (JORDAN 2017d).

In Erfüllung ihrer internationalen Funktionen präsentierten die Vertreter Österreichs die folgenden Papiere: Bericht des Koordinators der Toponymic Guidelines for Map and Other Editors for International Use (RAMPL 2017a), Bericht der UNGEGN Working Group on Exonyms (JoRDAN 2017e), Bericht der Joint ICA/IGU Commission on Toponymy (JoRDAN 2017f), Bericht über 50 Jahre DGSD (JORDAN et al. 2017) sowie eine Zusammenstellung weltweit einheitlicher Merkmale und Kriterien des Exonymengebrauchs - das Endergebnis mehrerer eigener Treffen der Working Group on Exonyms (JoRDAN 2017g).

Ähnliche Berichte über die Toponymic Guidelines for Map and Other Editors for International Use (RAmPL 2017b), die UNGEGN Working Group on Exonyms (JoRDAn 2017h) und die Joint ICA/ IGU Commission on Toponymy (JoRDAN 2017i) wurden auch in der UNGEGN-Sitzung vorgelegt.

\section{Umschrift des Arabischen}

Fachlich wichtigster Diskussionspunkt der Konferenz war der Vorschlag eines Transliterationssystems von der arabischen in die Lateinschrift, der von der Arabic Division kam, schon auf einigen Sitzungen der UNGEGN Working Group on Romanisation Systems, zuletzt in Prag [Praha] am 7. 
April 2017, intensiv diskutiert worden war und nun in der Konferenz maßgeblich von Saudi-Arabien und Jordanien vorgebracht wurde.

Er stellte eine Weiterentwicklung des sogenannten Beirut-Systems dar und sollte für das Standardarabische gelten. Die Schwierigkeit, eine einheitliche Umschrift für das Arabische einzuführen - diese Umschriftung also zu standardisieren - liegt darin, dass das Arabische eine defizitäre Silbenschrift ist, die nur die Konsonanten notiert, nicht auch die Vokale, weshalb es durch die unterschiedliche Einfügung von Vokalen zahlreiche regional gesprochene Varianten gibt, deren Lautgestalten eine einheitliche Umschrift oft nicht entsprechend wiederzugeben vermag.

Dies führte auch zu heftigem Widerstand vor allem Algeriens, unterstützt von Tunesien. Außer dem genannten Hauptargument wurde gegen den Vorschlag noch ins Treffen geführt, dass diese Umschrift in einigen Ländern Nordafrikas (Algerien, Tunesien, Ägypten) nicht verwendet werde und sie die Arabische Liga bisher nicht akzeptiert hätte. Die Gegenargumente der Befürworter, darunter das gewichtige Wort des Convenors der Working Group on Romanisation Systems Peeter Päll (Estland), es gehe nicht um die regional gesprochenen Varianten, sondern um das Standardarabische, und eine einheitliche Umschrift für dieses Metasystem sei als ein erster Schritt der Standardisierung besser als keine von den Vereinten Nationen empfohlene Umschrift, überzeugte aber schließlich doch die große Mehrheit der Delegationen. Nur Algerien, Tunesien und Ägypten stimmten gegen den Resolutionsentwurf und erwarben sich damit das Recht, die Umschrift nicht anzuwenden. Der Entwurf wurde inzwischen vom ECOSOC gebilligt und damit zu einer Resolution der Vereinten Nationen erhoben.

\section{4 „Special presentations“, Panel discussions, „side events“, Funktionswechsel und Ehrungen}

Den Verhandlungsgang im Plenum unterbrachen wohltuend 13 sogenannte „Special presentations“, zum großen Teil von auswärtigen Gästen, darunter dem Kartographen Tim TRAINOR (USA), Co-chair von UN-GGIM, zum Thema „United Nations Initiative on Global Geospatial Information Management“ und der Geographin Irena VASILIEv (USA) zu „New York City urban names“. Auch Gerhard RAMPL trug mit seinem Vortrag über „Field names in the Tyrol - collection, standardisation and cultural aspects" dazu bei.

Für eine starke Belebung des ,Konferenzalltags' sorgten auch die sechs Panel discussions im Plenum über aktuelle Themen, die auf Anregung der UNGEGN-Leitung von den jeweiligen Panel chairs konzipiert und organisiert worden waren. Eine davon zum Thema „United Nations resolutions on the reduction of exonyms: witnesses of the past or guidelines still observed?" leitete auch der Verfasser. Mit ihm diskutierten am Podium Helen Kerfoot (Kanada), Ferjan Ormeling (Niederlande), Kohei Watanabe (Japan) und Maciej Zych (Polen).

Neben dem Hauptstrang der Konferenz im Plenum, in den Morgenstunden vor Konferenzbeginn, während der Mittagspause und am Abend nach der Konferenz ereigneten sich noch etliche „Side events“. An den folgenden war der Verfasser leitend oder als Präsentator beteiligt: Business Meeting der UNGEGN Working Group on Exonyms; Präsentation der Joint ICA/IGU Commission on Toponymy; als Panelist in einem Treffen von einigen UNGEGN-Experten mit der UN Documentation Division. Zudem gab es - in einem ungezwungenen Rahmen und außerhalb des Hauptquartiers der Vereinten Nationen - zwei Treffen mit den Kollegen der Dutch- and German-speaking Division (DGSD, Abb. 4), eines davon auch gemeinsam mit der Norden Division.

Eine angenehme Abwechslung bedeutete auch die Einladung der österreichischen Vertretung bei den Vereinten Nationen zu einem Mittagessen mit der Ersten Botschaftssekretärin, Frau Mag. Andrea BACHER, die auch an der Arbeit der österreichischen Delegation regen Anteil nahm. 


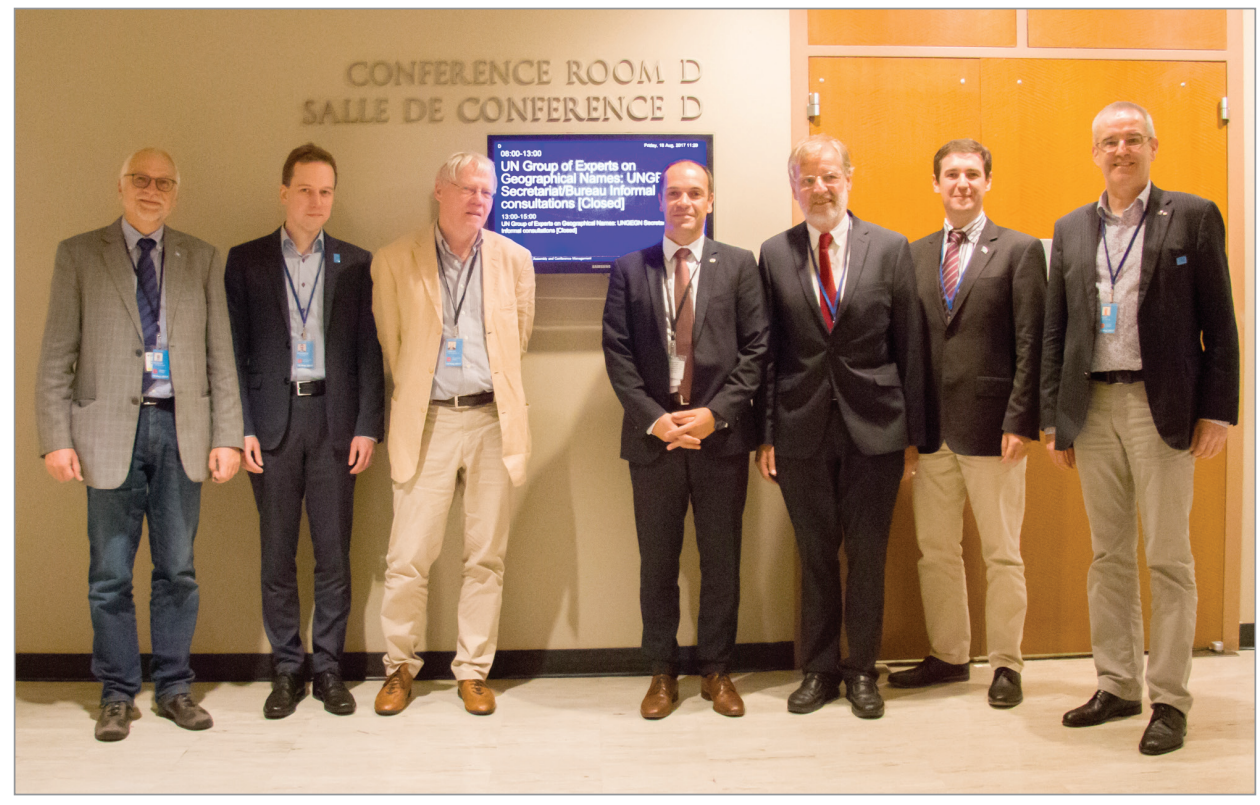

Foto: $\quad$ Maciej ZYCH 2017

Abb. 4: Die in New York anwesenden Mitglieder der DGSD: v.l.n.r. Bernd BeInsteIn (Deutschland), Jasper Hogerwerf (Niederlande), Ferjan Ormeling (Niederlande), Pier-Giorgio ZaCcheddu (DGSD-Vorsitzender, Deutschland), Peter JoRdan (Österreich), Gerhard RAmPL (Österreich), Jean-Yves PIRLot (Belgien)

Offenbar als Reaktion auf die großen Probleme, welche bei der Approbierung des Abschlussberichts bei der letzten UNGEGN-Sitzung in Bangkok [Krung Thep] im Jahr 2016 aufgetreten waren (Der Bericht konnte wegen beharrlicher Einsprüche am letzten Tag der Sitzung nicht mehr approbiert werden und wurde erst später nach komplizierten Verhandlungen gebilligt.) entschied man sich diesmal für einen Bericht, den man nur rudimentär nennen kann und der ohne auf Vortragsthemen und Diskussionsargumente einzugehen lediglich die Konferenzpapiere und Wortmeldungen nach Ländern notiert.

Die Konferenz, wohl verstärkt durch ihren zäsurhaften Charakter, war auch Anlass für zahlreiche Wechsel in den UNGEGN-Funktionen: So ging die Funktion des Convenors der Working Group on Toponymic Terminology von Staffan Nyström (Schweden) auf Trent PALMER (USA) über; die des Convenors der Working Group on Toponymic Training von Ferjan Ormeling (Niederlande) an Peder Gammeltoft (Dänemark); die des Convenors der Working Group on Exonyms von Peter Jordan (Österreich) an Kohei Watanabe (Japan).

In der Working Group on Romanisation Systems trat Catherine Cheetham (Vereinigtes Königreich) als Co-convenor an die Seite von Peeter Päll (Estland). Die Working Group on Pronunciation (Convenor: Tjeerd TRICHELAR, Niederlande) wurde aufgelöst; ihre Aufgaben wurden auf die Working Group on Romanisation Systems und die Working Group on Geographical Names as Cultural Heritage (Annette TöRENSJö, Schweden) aufgeteilt. An die Stelle des bisherigen UNGEGN Vice-chairs Ferjan Ormeling (Niederlande) trat Hasanuddin Abidin (Indonesien). Die Stelle des 2. 
Rapporteurs (neben Trent Palmer, USA) wurde anstelle von Peder Gammeltoft (Dänemark) mit Sungjae CHоо (Republik Korea) besetzt.

Den Abschluss der Konferenz bildete die Ehrung von fünf langjährigen und verdienten UNGEGN-Experten, die bei dieser Konferenz ihre Funktionen übergeben hatten oder schon früher aus Leitungsfunktionen geschieden waren und (wahrscheinlich) nicht mehr an UNGEGN-Sitzungen teilnehmen werden. Neben Helen Kerfoot (Kanada, Past UNGEGN Chair), Peter E. Raper (Südafrika, UNGEGN Chair vor KeRFoot), Ferjan ORMELING (Niederlande, UNGEGN Vice-chair, Vorsitzender der 11. Konferenz, langjähriger Convenor der UNGEGN Working Group on Toponymic Training) und Staffan Nyström (Schweden, langjähriger Convenor der Working Group on Toponymic Terminology) wurde diese Ehre auch dem Verfasser, v.a. für seine elfjährige Tätigkeit als Convenor der UNGEGN Working Group on Exonyms, zuteil (Abb. 5).

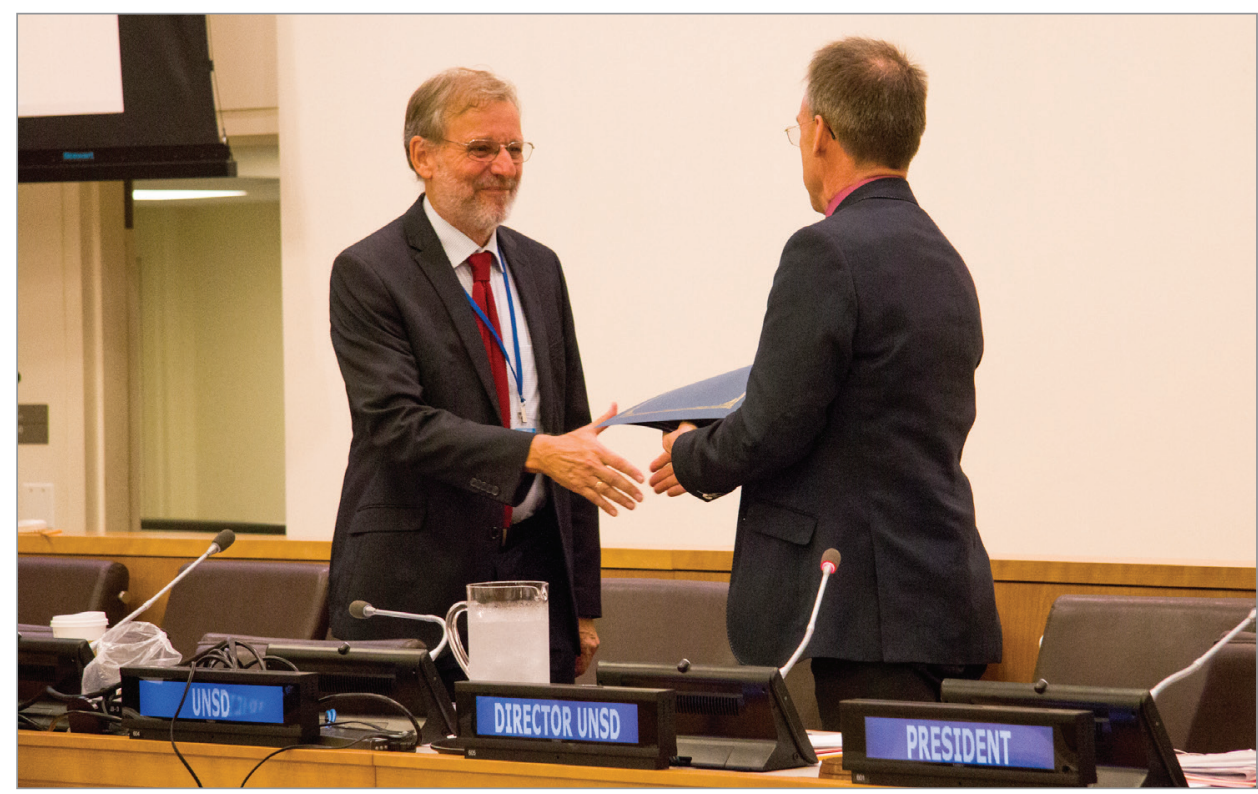

Foto: $\quad$ Maciej ZYCH 2017

Abb. 5: Stefan SchweInfest, Director, Statistics Division, Department of Economic and Social Affairs überreicht Peter JoRDAN die Ehrenurkunde

\section{Literaturverzeichnis}

BREU J. (1975), Geographisches Namenbuch Österreichs. Wien, Verlag der Österreichischen Akademie der Wissenschaften.

Cantile A., Kerfoot H. (Hrsg.) (2016), Place names as intangible cultural heritage. Firenze, IGMI. Jordan P. (2017a), Recommendations for the Naming of Urban Traffic Areas. Eleventh United Nations Conference on the Standardization of Geographical Names New York, 8-17 August 2017. E/CONF 105/21/CRP.21. New York, United Nations. 
JoRdAn P. (2017b), Exonym use in Austria. Eleventh United Nations Conference on the Standardization of Geographical Names New York, 8-17 August 2017. E/CONF 105/18/CRP.18. New York, United Nations.

Jordan P. (2017c), The endonym/exonym divide - Questions resolved and still open at the $15^{\text {th }}$ anniversary of the Working Group on Exonyms. Eleventh United Nations Conference on the Standardization of Geographical Names New York, 8-17 August 2017. E/CONF 105/17/ CRP.17. New York, United Nations.

Jordan P. (2017d), Report of Austria. Eleventh United Nations Conference on the Standardization of Geographical Names New York, 8-17 August 2017. E/CONF 105/22/CRP.22. New York, United Nations.

JoRdAn P. (2017e), Report of the Working Group on Exonyms. Eleventh United Nations Conference on the Standardization of Geographical Names New York, 8-17 August 2017. E/CONF $105 / 23$. New York, United Nations.

JoRDAN P. (2017f), Activities of the Joint International Cartographic Association/International Geographical Union Commission on Toponymy since 2012. Eleventh United Nations Conference on the Standardization of Geographical Names New York, 8-17 August 2017. E/CONF 105/19. New York, United Nations.

JoRDAn P. (2017g), Exonym use: Globally common characteristics and criteria. Eleventh United Nations Conference on the Standardization of Geographical Names New York, 8-17 August 2017. E/CONF 105/16/CRP.16. New York, United Nations.

Jordan P. (2017h), Report of the Working Group on Exonyms. United Nations Group of Experts on Geographical Names, Thirtieth session, New York, 7 and 18 August 2017, Working Paper No. 4. New York, United Nations.

JoRdAn P. (2017i), Joint ICA/IGU Commission on Toponymy. United Nations Group of Experts on Geographical Names, Thirtieth session, New York, 7 and 18 August 2017, Working Paper No. 10. New York, United Nations.

Jordan P., Ormeling F., Sievers J., Zaccheddu P.-G., Beinstein B. (2017), Fifty years of work by the Dutch- and German-speaking division of the United Nations Group of Experts on Geographical Names. Eleventh United Nations Conference on the Standardization of Geographical Names New York, 8-17 August 2017. E/CONF 105/79. New York, United Nations.

Piko-Rustia M. (2017), Slovene field and house names in Carinthia. Measures for the preservation and creative transfer of the Unesco intangible cultural Heritage. Eleventh United Nations Conference on the Standardization of Geographical Names New York, 8-17 August 2017. E/CONF 105/56/CRP.56. New York, United Nations.

RAMPL G. (2017a), Toponymic guidelines for map and other editors, for international use - some general remarks by the Coordinator. Eleventh United Nations Conference on the Standardization of Geographical Names New York, 8-17 August 2017. E/CONF 105/80/CRP.80. New York, United Nations.

Rampl G. (2017b), Toponymic guidelines for Map and Other Editors for International Use - Report of the Coordinator. United Nations Group of Experts on Geographical Names, Thirtieth session, New York, 7 and 18 August 2017, Working Paper No. 9. New York, United Nations.

United Nations Group of Experts on Geographical Names (UNGEGN) (Hrsg.) - https://unstats.un.org/UNSD/geoinfo/UNGEGN/default.html 